SLAC-PUB-8948

August 2001

physics:/0008026

\title{
Longitudinal Laser Shaping in Laser Wakefield Accelerators
}

\author{
Anatoly Spitkovsky (1) and Pisin Chen (2) \\ (1) Department of Physics, \\ University of California, Berkeley, CA 94720 \\ (2) Stanford Linear Accelerator Center, \\ Stanford University, Stanford, CA 94309
}

Submitted to Physical Review Letters

Stanford Linear Accelerator Center, Stanford University, Stanford, CA 94309

Work supported by Department of Energy contract DE-AC03-76SF00515. 


\title{
Longitudinal Laser Shaping in Laser Wakefield Accelerators
}

\author{
Anatoly Spitkovsky ${ }^{(1)}$ and Pisin Chen ${ }^{(2)}$ \\ (1) Department of Physics, University of California, Berkeley, CA 94720 \\ (2) Stanford Linear Accelerator Center, Stanford University, Stanford, CA 94309
}

(April 22, 2001)

\begin{abstract}
We study the energetics of wake excitation during the laser-plasma interaction in application to laser wakefield accelerators. We find that both the wake amplitude and the accelerating efficiency (transformer ratio) can be maximized by properly shaping the longitudinal profile of the driving laser pulse. The corresponding family of laser pulse shapes is derived in the nonlinear regime of laserplasma interaction. Such shapes provide theoretical upper limit on the magnitude of the wakefield and efficiency by allowing for uniform photon deceleration inside the laser pulse. We also construct realistic optimal pulse shapes that can be produced in finite-bandwidth laser systems.
\end{abstract}

52.75.Di, 52.40.Nk, 41.75.Lx, 52.40.Mj

Current plasma accelerators can be broadly subdivided into two classes - the laser driven scheme [1], and the electron beam driven scheme [2]. While the accelerating plasma waves excited in these two schemes are very similar, the physics of wakefield excitation is quite different between them. In the beam-driven Plasma Wakefield Accelerator (PWFA) the electron beam loses energy to the plasma through interaction with the induced electrostatic field, while in the laser-driven Laser Wakefield Accelerator (LWFA) energy loss occurs via photon redshift or deceleration [3]. This process is due to the presence of wake-induced stationary modulations in the refractive index of the plasma as seen in the laser comoving frame 泊. With the recent advances in laser technology, the creation of ultrashort pulses with virtually arbitrary temporal profiles is now possible by using amplitude and phase pulse shapers $[5$. Since perturbations to the refractive index of the plasma depend on pulse shape, different laser shapes will vary in their coupling to the plasma. A natural question therefore arises: what laser shape is the "optimal" for laser-plasma accelerators?

The number of parameters involved in selecting a particular pulse shape can be overwhelming. One can characterize a shape by the value of its total energy, length, maximum laser field, amplitude of plasma wake, etc., in addition to an infinite-dimensional space of actual shape functions. Luckily, not all of these parameters are independent or even well-defined. In this Letter we argue that the only two meaningful parameters that describe a laser shape from the stand point of wake excitation are the total pulse energy and its depletion length. Using these parameters different laser shapes can be consistently classified and cross-compared while desired properties such as wake amplitude or efficiency can be optimized.

Let us consider a homogeneous unmagnetized plasma which is charge neutral, at rest, and has initial density $n_{p}$ in the absence of electromagnetic wave. Laser propagates along the $z$ axis with initial frequency $\omega_{0} \gg \omega_{p} \equiv$ $\sqrt{4 \pi e^{2} n_{p} / m_{e}}$. In the laser comoving frame, the plasma response can be written in terms of the independent dimensionless variables $\zeta=k_{p}\left(z-v_{g} t\right)$ and $\tau=k_{p} c t$, where $k_{p}$ is the plasma wavenumber, and $v_{g} \approx-c$ is the laser group velocity (for convenience the laser is moving in the negative $z$ direction). Introducing dimensionless normalized scalar and vector potentials $\phi(\zeta)$ and $a(\zeta)$, the parallel and perpendicular electric fields are $E_{\|}=-\left(m c^{2} k_{p} / e\right) \partial \phi / \partial \zeta$ and $E_{\perp}=-(m c / e) \partial a / \partial t=$ $-\left(m c^{2} k_{p} / e\right) \partial a / \partial \zeta$. The wakefield generation equation can then be written as [6:7]:

$$
\frac{d^{2} x}{d \zeta^{2}}=\frac{n_{e}}{n_{p}}-1=\frac{1}{2}\left(\frac{1+a^{2}(\zeta)}{x^{2}}-1\right)
$$

where $n_{e}$ is the perturbed electron plasma density, $x \equiv$ $1+\phi$ is the modified electrostatic potential, and $a^{2}(\zeta)$ is the dimensionless laser intensity envelope averaged over fast oscillations. Prior to the arrival of the laser the normalized wakefield $\mathcal{E} \equiv e E_{\|} / m_{e} c \omega_{p}=-d x / d \zeta$ is zero. A formal solution for the electric field outside the laser can be written as the first integral of (1): $\left[\mathcal{E}^{\text {out }}(\zeta)\right]^{2}=-(x-1)^{2} / x+\int_{-\infty}^{\infty} a^{2} x^{\prime} / x^{2} d \zeta$, which reaches a maximum value at $x=1$ :

$$
\left[\mathcal{E}_{\text {max }}^{\text {out }}\right]^{2}=-\int_{-\infty}^{\infty} a^{2}(\zeta)\left(\frac{\partial}{\partial \zeta} \frac{1}{x}\right) d \zeta
$$

This expression can be understood in terms of the deposition of laser energy into plasma. Due to negligible scattering, the photon number in the laser is essentially constant, and the laser energy loss is predominantly in the form of frequency redshift, or photon deceleration [8,9]:

$$
\frac{\partial \omega}{\partial z}=-\frac{1}{2} \frac{\omega_{p}^{2}}{\omega} k_{p} \frac{\partial}{\partial \zeta} \frac{n_{e}}{\gamma n_{p}}=-\frac{\omega_{p}^{2}}{2 \omega} k_{p}\left(\frac{\partial}{\partial \zeta} \frac{1}{x}\right) .
$$

The energy density in the wake from (2) can then be interpreted as the intensity-weighted integral of the photon deceleration throughout the pulse. Let's denote the wake-dependent part of the photon deceleration function 
as $\kappa(\zeta) \equiv x^{\prime} / x^{2}$. It is closely related to the characteristic laser depletion length $l_{d}$, or the distance in which the maximally decelerated laser slice redshifts down to $\omega_{p}$ assuming a nonevolving wakefield. From (3), $l_{d}=$ $\left[\left(\omega_{0} / \omega_{p}\right)^{2}-1\right] / k_{p} \kappa_{\max }$, where $\kappa_{\max }$ is the maximum of $\kappa(\zeta)$ inside the laser. The value of the peak wakefield in (2) is bounded from above by the total laser energy and the maximum photon deceleration:

$$
\left[\mathcal{E}_{\text {max }}^{\text {out }}\right]^{2}=\int_{-\infty}^{\infty} a^{2}(\zeta) \kappa(\zeta) d \zeta \leq \kappa_{\max } \int_{-\infty}^{\infty} a^{2}(\zeta) d \zeta
$$

One possible optimization problem can then be formulated as follows: given some fixed laser energy $\varepsilon_{0}$ (i.e., the integral of $a^{2}$ ), and the desired depletion length (i.e., $\kappa_{\max }=\kappa_{0}$ ), what laser shape would produce the largest possible wakefield? From (4) it is clear that such a shape should maintain a constant maximal photon deceleration throughout the pulse. If the laser is present for $\zeta>0$, then in order to satisfy the boundary conditions the photon deceleration should rise from 0 to value $\kappa_{0}$ at the very beginning of the pulse, e.g., like a step-function: $\kappa(\zeta)=\kappa_{0} \theta\left(\zeta^{+}\right)$. Here, $\zeta^{+} \equiv \zeta-0^{+}$in order to avoid ambiguities with the values of step-function at 0 . The corresponding laser profile is then found from the wake equation (11):

$$
a_{l}^{2}(\zeta)=\frac{2 \kappa_{0} \delta\left(\zeta^{+}\right)}{\left(1-\kappa_{0} \zeta\right)^{4}}+\frac{4 \kappa_{0}^{2} \theta^{2}\left(\zeta^{+}\right)}{\left(1-\kappa_{0} \zeta\right)^{5}}+\frac{1}{\left(1-\kappa_{0} \zeta\right)^{2}}-1,
$$

where $\zeta \in\left[0, \zeta_{f}<1 / \kappa_{0}\right]$, and $\delta\left(\zeta^{+}\right)$is a delta-function such that $\int_{0}^{\zeta>0} \delta\left(y^{+}\right) d y=1$. A schematic drawing of the optimal laser intensity variation and its associated plasma wakefield are shown in Fig. 11. Generally, the shape consists of a $\delta$-function at the front followed by a ramp in intensity which is cut off at $\zeta_{f}$. In the linear regime, when $a^{2} \ll 1, \kappa_{0} \rightarrow 0$, the ramp reduces to a triangular shape found in [9,10]: $a^{2}=2 \kappa_{0}\left(\delta\left(\zeta^{+}\right)+\zeta\right)$. We note that (5) describes a family of shapes, rather than a fixed shape. The actual profile of the optimal pulse depends on the deceleration parameter $\kappa_{0}$ set by the desired depletion length and the pulse length $\zeta_{f}$, which is determined from the available total energy:

$$
\varepsilon_{0}=2 \kappa_{0}+\frac{\zeta_{f}\left[\kappa_{0}^{2}+\left(1-\kappa_{0} \zeta_{f}\right)^{3}\right]}{\left(1-\kappa_{0} \zeta_{f}\right)^{4}} .
$$

Although the pulse length cannot exceed $\zeta_{c} \equiv 1 / \kappa_{0}$, the rise of $a^{2}$ towards the end of the pulse guarantees that any finite laser energy can be accommodated for $\zeta_{f}<\zeta_{c}$. The two terms in (6) represent the energy contained in the $\delta$-function precursor and the main pulse. It is clear that for a fixed total energy there exists a maximum value of $\kappa_{0}=\varepsilon_{0} / 2$ which is achieved when $\zeta_{f} \rightarrow 0$, i.e., all of the energy is concentrated in the $\delta$-function. This shape, which is a particular case of the general optimal shape (5), excites the largest possible wakefield and has the smallest depletion length among all pulses of fixed energy. For circularly polarized pulses with cylindrical transverse crossection of radius $r_{0}$ and wavelength $\lambda$, the maximum achievable wake is then given by:

$$
E_{\max }=6.54 E_{w b}\left[\frac{U_{0}}{1 \mathrm{~J}}\right]\left[\frac{\lambda}{1 \mu \mathrm{m}}\right]^{2}\left[\frac{10 \mu \mathrm{m}}{\mathrm{r}_{0}}\right]^{2}\left[\frac{n_{p}}{10^{18} \mathrm{~cm}^{-3}}\right]^{1 / 2}
$$

where $U_{0}$ is the total pulse energy (in Joules) and $E_{w b}=$ $96\left[n_{p} / 10^{18} \mathrm{~cm}^{-3}\right] \mathrm{GV} / \mathrm{m}$ is the nonrelativistic wavebreaking field.

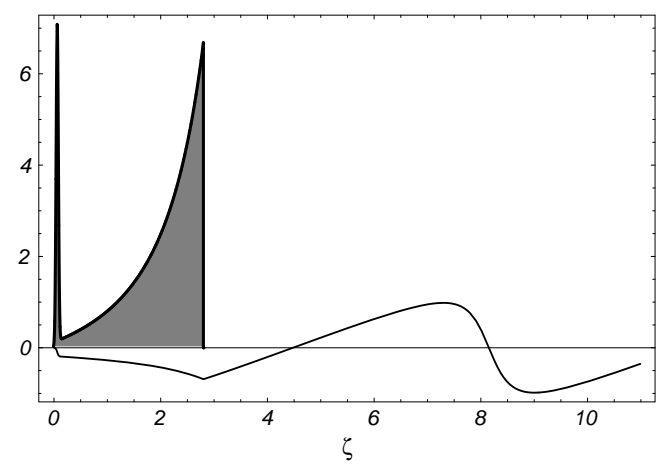

FIG. 1. General shape of the nonlinear optimal laser intensity profile and its corresponding wakefield (arbitrary units).

The method used for obtaining the optimal shape (5) is actually more general and can be used to determine laser shapes that generate other variations in the nonlinear index of refraction. Having a physical requirement for the refractive index $\eta \equiv\left[1-\left(\omega_{p} / \omega\right)^{2} n_{e} / \gamma n_{p}\right]^{1 / 2}=$ $\left[1-\left(\omega_{p} / \omega\right)^{2} 1 / x\right]^{1 / 2}$, which for this case is the requirement of uniformity of photon deceleration, provides a constraint on $x(\zeta)$, which can then be used to find the laser shape from the wake equation. Alas, such a "reverse" solution is not always guaranteed to yield a physical (i.e., positive) $a^{2}(\zeta)$, so, in general, caution is advised.

While the generation of large accelerating gradients is a prerequisite for a successful accelerating scheme, the efficiency of acceleration should also be considered. For an accelerating scheme that involves transfer of energy from the driver beam to the accelerating beam, efficiency is measured in terms of the transformer ratio, or the ratio of the maximum rate of energy gain per particle of accelerating beam to the maximum rate of energy loss per particle of the driving beam. In the case of laserplasma accelerators, where the driving and accelerating beams consist of particles of different species, the following kinematic definition is more useful:

$$
R \equiv \frac{\left|\partial \gamma_{a} / \partial z\right|_{\max }}{\left|\partial \gamma_{d} / \partial z\right|_{\max }}
$$

where $\gamma_{d}$ and $\gamma_{a}$ are Lorentz factors for the driving and accelerating beams. In LWFA the particles in the trailing 
electron bunch are accelerated via electrostatic interaction with the wake, so $\left|\partial \gamma_{a} / \partial z\right|_{\max }=\left|e E_{\|}^{\max }\right| / m_{e} c^{2}=$ $k_{p}\left|\mathcal{E}_{\text {max }}^{\text {out }}\right|$. For the laser propagating in plasma $\gamma_{d} \approx \omega / \omega_{p}$,

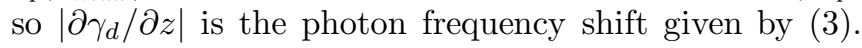
The transformer ratio for LWFA is then:

$$
R^{\mathrm{LWFA}}=\frac{2 \omega}{\omega_{p}} \frac{|\partial x / \partial \zeta|_{\max }^{\text {out }}}{|\partial(1 / x) / \partial \zeta|_{\text {max }}^{\text {in }}} \propto|\mathcal{E}|_{\text {max }}^{\text {out }} k_{p} l_{d}
$$

It follows from this definition that the transformer ratio is not only a measure of local accelerating efficiency, but is also directly related to the maximum energy that can be transferred to the accelerating beam particle over the laser depletion length (assuming no evolution of the wake during propagation).

As there is no absolute maximum of $R$, we can only look for a shape that maximizes $R$ subject to constraints. For instance, among the pulses of fixed energy and depletion length $R$ is maximized by a pulse that produces the largest wakefield. But this is precisely the optimal shape found above. A more general proof involves considering all pulses irrespective of total energy that create a given value of wakefield. It can then be shown [11] that a pulse which has the largest depletion length among these must maintain a constant photon deceleration inside the pulse, which again points to the shape (5). From (9) the optimal transformer ratio is then:

$$
R^{\mathrm{LWFA}}=\frac{2 \omega}{\omega_{p}} \sqrt{\frac{1+\left(k_{p} L_{p}\right)^{2}\left[1-\kappa_{0}\left(k_{p} L_{p}\right)\right]^{3}}{\left[1-\kappa_{0}\left(k_{p} L_{p}\right)\right]^{4}}}
$$

where $L_{p}=\zeta_{f} / k_{p}$ is the pulse length. In the linear regime optimal transformer ratios for both LWFA and PWFA schemes scale identically with the pulse/beam length: $R^{\mathrm{LWFA}} \rightarrow\left(2 \omega / \omega_{p}\right) \sqrt{1+\left(k_{p} L_{p u l s e}\right)^{2}}, R^{\mathrm{PWFA}} \rightarrow$ $\sqrt{1+\left(k_{p} L_{\text {beam }}\right)^{2}}$ [12]. The LWFA scheme is intrinsically more efficient by a factor of $2 \omega / \omega_{p}$, which is needed for viability of LWFA since lasers are typically "slower" drivers than electron beams.

The advantage of using the optimal pulse shape is best seen in comparison with the unshaped (Gaussian) pulse. For a given Gaussian pulse (or any other non-optimal shape) one can always construct a corresponding optimally shaped pulse with the same laser energy such that the photon deceleration across the optimal pulse equals to the peak photon deceleration in the unshaped one (i.e., both pulses have equal depletion lengths). Unshaped pulses deplete first in the region where photon deceleration is the largest, whereas a laser with the optimal shape loses all its energy in a depletion length due to uniform photon deceleration, thus enhancing instantaneous energy deposition and wakefield. For a numerical example, we consider the optimal and Gaussian pulses of total energy $0.5 \mathrm{~J}$, wavelength $1 \mu \mathrm{m}$ and cylindrical radius $10 \mu \mathrm{m}$ in a plasma with $n_{p}=10^{18} \mathrm{~cm}^{-3}$. The transformer ratio, the maximum wakefield, the required pulse length, and the corresponding peak $a_{0}$ are shown in Fig. 2 as functions of depletion length.

From Fig. 2 we see that the transformer ratio and the maximum wakefield are consistently larger for shaped pulses. In fact, the lines for optimal pulse wakefield and transformer ratio represent theoretical upper limits for all pulses of given energy. The Gaussian pulse achieves a maximum transformer ratio when its length (measured here as FWHM) equals $1 / 2$ of the relativistic plasma wavelength. The effects of shaping are especially prominent for longer pulses, where Gaussian pulse yields almost no wake excitation due to plasma oscillations inside the pulse that cause part of the laser photons to absorb energy from the wake. On the other hand, a shaped laser postpones plasma oscillation until the end of the pulse, and all photons decelerate uniformly. For very short pulses, the differences between the two shapes are minimal. This is due to the fact that very short Gaussian pulses of fixed energy asymptotically approach the delta-function limit of the short optimal shape.
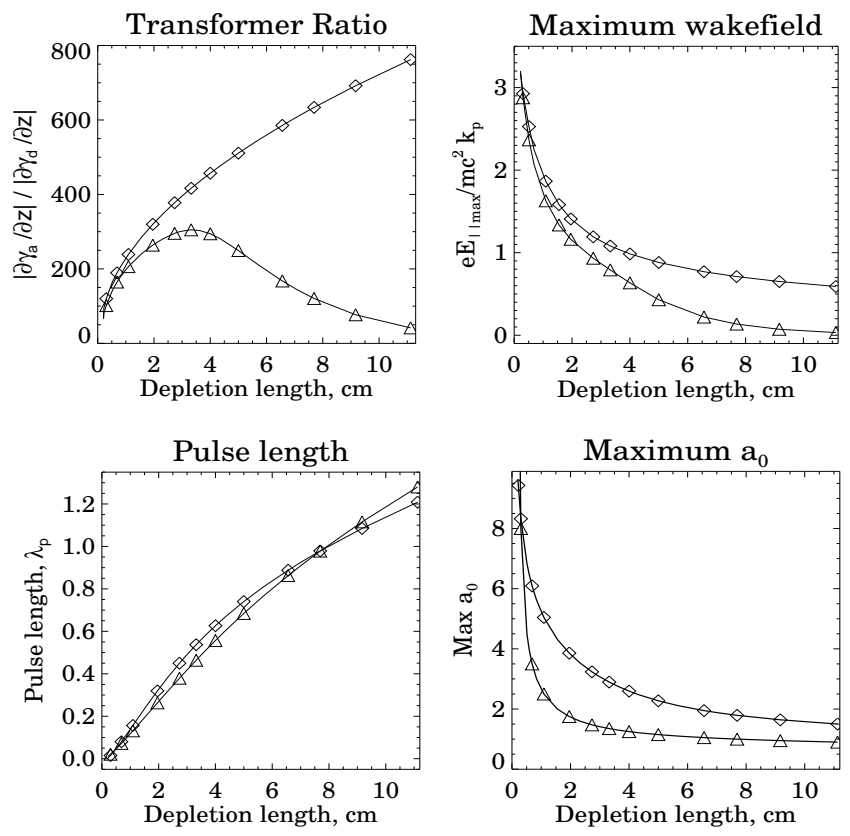

FIG. 2. Comparison of the transformer ratio, maximum wakefield, pulse length, and maximum normalized vector potential in shaped (diamonds) and Gaussian (triangles) pulses of equal depletion lengths and constant pulse energy of $0.5 \mathrm{~J}$.

Although short pulses generally produce the largest wakefields, their efficiency is close to minimal possible, as the depletion length decreases faster than increase in the wake. Therefore, the choice of the appropriate pulse shape for LWFA stage will depend on specific experimental conditions. If the laser-plasma interaction distance is limited by instabilities, diffraction or dephasing, then in order to maximize the electron energy gain one should try to achieve the largest accelerating gradient, which can be accomplished with ultrashort pulses. If, how- 
ever, the interaction length is less constrained, such as the case for propagation in plasma channels [13], then using a finite-length shaped pulse will result in a greatly improved overall energy gain per stage. An added benefit of pulse shaping is the suppression of modulational instability that affects unshaped pulses that are longer than plasma wavelength. When all photons redshift, or "slow down", at the same rate, different laser slices do not overrun each other, and the 1D laser self-modulation is suppressed.
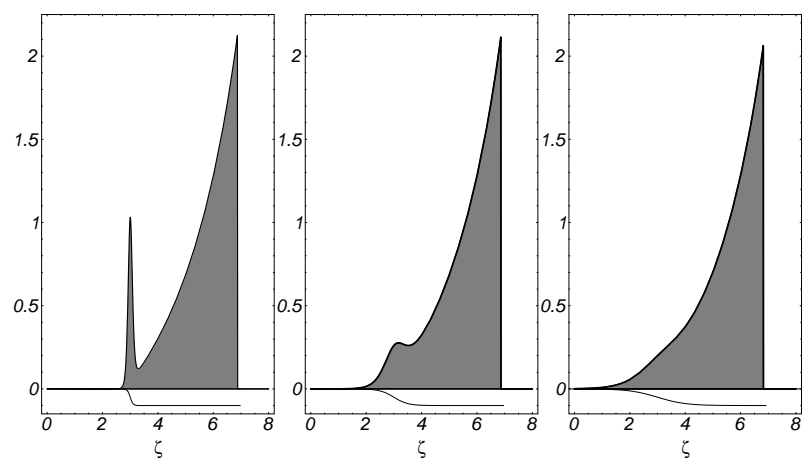

FIG. 3. Laser intensity (shaded) and the associated photon deceleration $(-\kappa(\zeta))$ for pulses of the same total energy and characteristic depletion length in the order of decreasing $\alpha$.

As the optimal pulse shape is associated with a deltafunction precursor, the feasibility of such a structure may be a concern. We note that the purpose of this precursor is to bring the photon deceleration from zero in the quiescent plasma before the laser to a finite value $\kappa_{0}$ at the beginning of the main pulse. This can also be achieved with a more physical prepulse, whose shape can be found once a smooth function $\kappa(\zeta)$ is chosen. For our example we choose $\kappa(\zeta)=\kappa_{0}\left[1+\tanh \alpha\left(\zeta-\zeta_{0}\right)\right] / 2$, where $\alpha$ is a steepness parameter and $\zeta_{0}$ is an arbitrary offset. The corresponding laser shape is then:

$$
a^{2}=\frac{\kappa_{0} \alpha \operatorname{sech}^{2} \alpha \zeta_{1}}{\chi^{4}\left(\zeta_{1}\right)}+\frac{\kappa_{0}^{2}\left[1+\tanh \alpha \zeta_{1}\right]^{2}}{\chi^{5}\left(\zeta_{1}\right)}+\frac{1}{\chi^{2}\left(\zeta_{1}\right)}-1,
$$

where $\zeta_{1} \equiv \zeta-\zeta_{0} \leq \zeta_{f}$ and $\chi\left(\zeta_{1}\right) \equiv 1+\left(\kappa_{0} / 2 \alpha\right) \ln \frac{1}{2}-$ $\kappa_{0}\left[\zeta_{1}+\frac{1}{\alpha} \ln \left(\cosh \alpha \zeta_{1}\right)\right] / 2$. As before, the pulse length $\zeta_{f}$ can be found from the available pulse energy. For a stepfunction photon deceleration $(\alpha \rightarrow \infty)$ expression (11) asymptotes to equation (5). However, for finite values of $\alpha$ the delta-function precursor spreads out and can even disappear as shown in Fig. 3. The family of shapes given by (11) is better suited for the finite-bandwidth laser systems that have a lower limit on achievable feature size. The values of the maximum wakefield for pulses in Fig. 3 are within few percent of the value for an equivalent delta-function optimal pulse because the bulk of the modified laser pulse still experiences constant maximal photon deceleration. The wakefield further degrades with longer rise times of $\kappa(\zeta)$. It is also possible to construct optimal shapes that propagate in a pre-existing plasma oscillation and act as wakefield amplifiers [11]. Such shapes also do not require delta-function precursors.

Several issues should be addressed before the laser pulse shaping concept can be fully utilized. Even without the delta-function precursor, the finite laser bandwidth will necessarily smooth out sharp rises and falls of the optimal pulse shape. Although we do not anticipate adverse effects when the feature size is much smaller than the plasma wavelength, the 1D self-consistent laser evolution and stability of realistic optimal shapes are currently under investigation. Another consideration is the influence of the laser-plasma interaction in the transverse dimension on the evolution of the pulse. Many of the laser-plasma instabilities are seeded by the wakefieldinduced perturbations of the index of refraction. As we have demonstrated in this Letter, the nonlinear index of refraction can be effectively controlled through laser shaping, thus suggesting the method of delaying the onset of these instabilities. Whether this approach increases the growth rates of other instabilities, particularly in the transverse dimension, remains to be investigated.

We would like to thank J. Arons, A. Charman, T. Katsouleas, W. B. Mori, and J. Wurtele for fruitful discussions and suggestions.

[1] T. Tajima, J. M. Dawson, Phys. Rev. Lett. 43, 267 (1979).

[2] P. Chen, J. M. Dawson, R. Huff, T. Katsouleas, Phys. Rev. Lett. 54, 693 (1985).

[3] S. Wilks, J. M. Dawson, W. B. Mori, T. Katsouleas, M. Jones, Phys. Rev. Lett. 62, 2600 (1989).

[4] W. B. Mori, IEEE J. Quant. Elec. 33, 1942 (1997)

[5] F. Verluise, V. Laude, Z. Cheng, et al., Optics Lett. 25, 575 (2000).

[6] P. Sprangle, E. Esarey, J. Krall, and G. Joyce, Phys. Rev. Lett. 69, 2200 (1992).

[7] E. Esarey, P. Sprangle, J. Krall, A. Ting, IEEE Trans. Plasma Sci. 24, 252 (1996).

[8] E. Esarey, A. Ting, and P. Sprangle, Phys. Rev. A 42, 3526, (1990).

[9] P. Chen, A. Spitkovsky, AIP Conf. Proc. 472, 321 (1999).

[10] P. Chen, A. Spitkovsky, T. Katsouleas, W. B. Mori, Nucl. Instr. Meth. 410, 488 (1998).

[11] A. Spitkovsky, P. Chen, AIP Conf. Proc. Advanced Accelerator Concepts 2000, in press.

[12] P. Chen, J. J. Su, J. M. Dawson, K. Bane, and P. Wilson, Phys. Rev. Lett. 56, 1252 (1986).

[13] E. Esarey, P. Sprangle, J. Krall, A. Ting, G. Joyce, Phys. Fluids B 5, 2690 (1993). 